\title{
Análisis del estado actual de certificaciones CMMI- DEV ver. 1.3 año 2013 y 2014, a nivel Mundial y en México
}

\author{
Yesenia Nohemí González Meneses, Nayely Yaline León Padilla, José \\ Juan Hernández Mora, María Guadalupe Medina Barrera \\ Instituto Tecnológico de Apizaco, Departamento de Sistemas y \\ Computación, Apizaco, Tlaxcala \\ yeseniaglez@hotmail.com, salome31_7@hotmail.com, jihmora@msn.com, \\ lupita_medina@hotmail.com
}

\begin{abstract}
Resumen. En este artículo, se presenta un análisis de las organizaciones desarrolladoras de software que a nivel mundial han sido evaluadas de acuerdo al modelo CMMI, en el apartado de CMMI-Dev en su versión 1.3 en los años 2013 y 2014. Este trabajo da como resultado el ranking mundial por país en cuanto a certificaciones en CMMI-DEV ver. 1.3 en sus cinco niveles de madurez, lo que refleja el interés de que las empresas desarrolladoras de software tienen en mejorar la calidad en sus procesos para el desarrollo de software y lo importante que en la actualidad es el hecho de obtener una certificación avalada internacionalmente. También se presenta un estudio enfocado a las empresas mexicanas que lograron certificarse en CMMI, en estos mismos años, apoyadas en algunos casos por programas gubernamentales que están impulsando la certificación internacional a través de la promoción de convocatorias que les ofrecen diferentes apoyos, encaminados al mejoramiento de la calidad de sus procesos.
\end{abstract}

Palabras clave: CMMI-DEV, mejora de procesos, certificaciones de calidad, desarrollo de software.

\section{Introducción}

Hoy en día, el desarrollo de software con calidad es una necesidad que las empresas desarrolladoras de software tienen, demandado por sus mismos clientes, que esperan que se les garantice la calidad en sus productos y que a la vez cumplan con especificaciones internacionales. Por muy pequeñas que estas empresas sean, no se pueden dar el lujo de sacrificar la calidad, existe una alta competencia de industrias extranjeras como las de los 
países de India, China y EEUU, debido a que estos países tienen altos índices de certificaciones de calidad y mejora de procesos, lo que les permite garantizar a sus clientes la calidad de sus productos.

La competitividad de las empresas debe basarse en mejoras e innovación de sus procesos, que brinden una propuesta de valor a sus clientes. Es por ello que las empresas buscan una alternativa en la mejora continua de sus procesos clave mediante una estrategia de calidad que incluya la implantación de algún modelo o estándar de calidad formal y reconocido, como es el modelo CMMI (Capability Maturity Model Integration).

\section{Breve historia de CMMI}

El Instituto de Ingeniería de Software (SEI, Software Engineering Institute), fue fundado en el año de 1984 mediante el financiamiento del Departamento de Defensa de Estados Unidos, es administrado por la Universidad de Canergie Mellon. Este instituto fue creado con el objetivo de desarrollar modelos de evaluación y mejora en el desarrollo de software, que dieran solución a los problemas de planeación y desarrollo de software, durante la construcción de sistemas militares del ejército de Estados Unidos.

Desde su creación el SEI, se ha centrado en proporcionar la base para mejorar el desarrollo de software, basado sobre el principio de que la calidad del producto depende principalmente de la calidad de los procesos empleados en su desarrollo y considerando a las tareas de desarrollo del software como una serie de procesos que se puedan definir, medir y controlar [1].

El SEI ha creado un conjunto de modelos utilizados para incrementar la calidad del software, entre los cuales se encuentran CMMI-DEV, CMMI-ACQ, CMMI-SVC, SCAMPI E IDEAL. Los modelos de CMMI son colecciones de buenas prácticas que ayudan a las organizaciones a mejorar sus procesos [1].

\subsection{Marco CMMI}

El marco CMMI brinda la organización necesaria para establecer los modelos del CMMI y los componentes de evaluación de CMMI. El marco CMMI permite el uso de múltiples modelos, para ello aloja componentes comunes a todos los modelos CMMI aplicables a cualquier modelo. El material común es nombrado "CMMI Model Foundation" o "CMF", los componentes del CMF son utilizados para conformar todos los modelos generados a partir del marco CMMI, esos componentes son armonizados con el material aplicable a un área de interés para crear un modelo. La colección de componentes que son utilizados para construir modelos, materiales de entrenamiento y materiales en el área de interés son llamados "constelación".

Las constelaciones que han sido definidas para CMMI actualmente son tres [2]: 
+ CMMI para el desarrollo (CMMI-DEV), brinda las guías necesarias para medir, controlar y gestionar los procesos de desarrollo.

+ CMMI para la adquisición (CMMI-ACQ), brinda una guía para controlar y gestionar la adquisición de productos y servicios que cumplan con las necesidades del cliente.

+ CMMI para los servicios (CMMI-SVC), brinda guías para aquellos que proveen servicios dentro de las organizaciones y a clientes externos.

\section{CMMI-DEV}

El modelo CMMI para Desarrollo, brinda un conjunto de guías completas e integradas para desarrollar productos y servicios, además de brindar orientación para aplicar las buenas prácticas CMMI en una organización de desarrollo. Las buenas prácticas del modelo se centran en las actividades para desarrollar productos y servicios de calidad con el objetivo de cumplir las necesidades de clientes y usuarios finales [2].

CMMI-DEV contiene 22 áreas de proceso [2], un área de proceso es un conjunto de prácticas que se relacionan y que cuando se implementan en conjunto satisfacen una serie de metas consideradas importantes para mejorar esa área.

Las 22 áreas de proceso se presentan a continuación por orden alfabético de sus acrónimos en inglés [3]:

1. Análisis Causal y Resolución (CAR).

12. Monitorización y Control del Proyecto (PMC).

2. Gestión de Configuración (CM).

13. Planificación del Proyecto (PP).

3. Análisis de Decisiones y Resolución (DAR).

14. Aseguramiento de la Calidad del Proceso y del Producto (PPQA).

4. Gestión Integrada del Proyecto (IPM).

15. Gestión Cuantitativa del Proyecto (QPM).

5. Medición y Análisis (MA).

16.Gestión de Requisitos (REQM).

6. Definición de Procesos de la Organización (OPD).

17. Desarrollo de Requisitos (RD).

7. Enfoque en Procesos de la Organización (OPF).

18. Gestión de Riesgos (RSKM).

8. Gestión del Rendimiento de la Organización (OPM).

19. Gestión de Acuerdos con Proveedores (SAM).

9. Rendimiento de Procesos de la Organización (OPP).

20. Solución Técnica (TS).

10. Formación en la Organización (OT).

21. Validación (VAL).

11. Integración del Producto (PI).

22. Verificación (VER) 


\section{Objetivo y alcance del estudio}

El objetivo de este trabajo es presentar un análisis de la información estadística de organizaciones que han sido evaluadas por el CMMI Institute, en la constelación de CMMI-DEV versión 1.3 en los años 2013 y 2014.

En la página oficial del CMMI Institute en "Published Apraisal Results" [4] se encuentran publicados los resultados de las evaluaciones realizadas a las organizaciones que implantaron algún nivel de CMMI, en cualquiera de sus constelaciones y versiones (ya sea versión 1.2 y 1.3). En esta página se pueden consultar resultados por modelo/constelación, nivel de madurez, año (que va desde el año 2011 hasta los resultados más actuales de 2014) y país.

Este trabajo se centrará en los resultados de las evaluaciones de 2013 en CMMI-DEV ver. 1.3, debido a que son los datos más actuales y completos hasta el momento. También se analizaran de una manera breve los cuatro primeros lugares en certificaciones en lo que va del año 2014.

\section{Análisis estadístico}

Al finalizar el año 2013, existen 1389 certificaciones activas en CMMI- DEV ver 1.3 alrededor del mundo, estas certificaciones están repartidas en 53 países. Debido a que existen diferentes tipos de constelaciones, algunas empresas pueden tener más de una certificación activa, también se da el caso de que existan diversas certificaciones compartidas entre diferentes países, un ejemplo de ello es la organización Tata Consultancy Services Limited (TCS) que está presente en los siguientes países: Argentina, India, Brasil, China, Colombia, Estados Unidos, Paraguay, Chile, Ecuador, México y Uruguay. En este estudio la certificación cuenta por separado para cada país debido a que la certificación incluye una evaluación por país de manera independiente [4].

A continuación en la Tabla 1, se muestran el número total de certificaciones obtenidas por país, divididas por niveles de madurez.

Tabla 1. Listado de certificaciones CMMI-DEV ver 1.3 año 2013, por país.

\begin{tabular}{lllllll}
\hline Núm. & País & Nivel 5 & Nivel 4 & Nivel 3 & Nivel 2 & $\begin{array}{l}\text { Total por } \\
\text { País }\end{array}$ \\
\hline 1 & China & 31 & 26 & 558 & 6 & 621 \\
2 & EEUU & 20 & 2 & 157 & 72 & 251 \\
3 & India & 36 & 0 & 115 & 4 & 155 \\
4 & México & 4 & 1 & 27 & 17 & 49 \\
5 & Brasil & 3 & 1 & 18 & 14 & 36 \\
\hline
\end{tabular}




\begin{tabular}{|c|c|c|c|c|c|c|}
\hline 6 & España & 4 & 0 & 10 & 21 & 35 \\
\hline 7 & $\begin{array}{l}\text { República } \\
\text { de Corea }\end{array}$ & 2 & 2 & 16 & 11 & 31 \\
\hline 8 & Japón & 0 & 5 & 14 & 4 & 23 \\
\hline 9 & Francia & 0 & 0 & 6 & 12 & 18 \\
\hline 10 & Colombia & 5 & 0 & 9 & 2 & 16 \\
\hline 11 & Turquía & 1 & 0 & 11 & 1 & 13 \\
\hline 12 & Tailandia & 2 & 0 & 6 & 3 & 11 \\
\hline 13 & Taiwán & 0 & 1 & 9 & 1 & 11 \\
\hline 14 & Italia & 0 & 0 & 7 & 3 & 10 \\
\hline 15 & Argentina & 2 & 0 & 4 & 3 & 9 \\
\hline 16 & Perú & 0 & 0 & 8 & 1 & 9 \\
\hline 17 & Canadá & 1 & 0 & 4 & 3 & 8 \\
\hline 18 & Portugal & 2 & 0 & 3 & 3 & 8 \\
\hline 19 & Vietnam & 2 & 0 & 5 & 0 & 7 \\
\hline 20 & Alemania & 0 & 0 & 2 & 4 & 6 \\
\hline 21 & Chile & 2 & 0 & 1 & 3 & 6 \\
\hline 22 & Egipto & 1 & 0 & 3 & 1 & 5 \\
\hline 23 & Australia & 1 & 0 & 2 & 1 & 4 \\
\hline 24 & Filipinas & 3 & 0 & 1 & 0 & 4 \\
\hline 25 & Malaysia & 1 & 0 & 3 & 0 & 4 \\
\hline 26 & Reino Unido & 0 & 0 & 3 & 1 & 4 \\
\hline 27 & Bélgica & 0 & 0 & 1 & 2 & 3 \\
\hline 28 & Pakistán & 0 & 0 & 2 & 1 & 3 \\
\hline 29 & Singapur & 1 & 0 & 2 & 0 & 3 \\
\hline 30 & Hong Kong & 1 & 0 & 0 & 1 & 2 \\
\hline 31 & Luxemburgo & 0 & 0 & 1 & 1 & 2 \\
\hline 32 & Países bajos & 0 & 0 & 0 & 2 & 2 \\
\hline \multirow[t]{2}{*}{33} & Rusia & 2 & 0 & 0 & 0 & 2 \\
\hline & $\begin{array}{c}\text { Total por } \\
\text { Nivel }\end{array}$ & 132 & 38 & 1016 & 205 & 1389 \\
\hline
\end{tabular}

Como se puede apreciar en la Tabla 1, el país con mayor número de certificaciones totales es China con un total de 621, le siguen Estados Unidos con 251, India con 155, México con 49 y Brasil con 36 certificaciones. Los países que solo obtuvieron una certificación en el año 2013 en CMMI-DEV ver. 1.3, aunque no aparecen listados en la tabla por cuestiones de espacio fueron: Arabia, Austria, Bangladesh, Bulgaria, Chipre, 
Ecuador, Grecia, Indonesia, Israel, Jordán, Kenia, Kuwait, Letonia, Polonia, Sir Lanka, Suecia, Suiza, Paraguay, Romania y Uruguay.

Con lo que respecta al número de certificaciones en el Nivel 5 de madurez, existen 132 a nivel mundial, el país que ocupa el primer lugar en lo que se refiere a certificaciones en este nivel es India con 36, en segundo lugar se encuentra China con 31, Estados Unidos ocupa la tercera posición con 20 certificaciones, en cuarto lugar se encuentra Colombia con 5 certificaciones y en la quinta posición se encuentran México y España con 4 certificaciones, en la sexta posición se encuentra Brasil y Filipinas con 3 certificaciones, en séptimo lugar se encuentran República de Corea, Tailandia, Argentina, Portugal, Vietnam, Chile y Rusia con 2 certificaciones y los países que cuentan con una certificación son Paraguay, Uruguay, Ecuador, Romania, Israel, Egipto, Australia, Canadá, Turquía, Singapur y Hong Kong [4]. Esta información se lista en la Tabla 2.

Tabla 2. Listado de certificaciones en el nivel 5 de CMMI-DEV ver. 1.3 año 2013, por país.

\begin{tabular}{c|cc}
\hline Posición & País & $\begin{array}{c}\text { Certificaciones en } \\
\text { Nivel 5 }\end{array}$ \\
\hline 1 & India & 36 \\
2 & China & 31 \\
3 & EEUU & 20 \\
4 & Colombia & 5 \\
5 & México & 4 \\
5 & España & 4 \\
\hline
\end{tabular}

Una de las empresas más importantes a nivel mundial es Tata Consultancy Services Limited, México, que es parte fundamental de TCS Latinoamérica, y es brazo de negocios de TCS que opera a lo largo de toda la región latinoamericana, con Centros de Entrega Global (GDC), en Argentina, Brasil, Chile, Colombia, Ecuador, Perú y Uruguay en el nivel 5 de CMMI, el estándar de calidad más alto de la industria de software; cuenta con centros de servicios de tercerización de procesos de negocios en Chile y Uruguay.

Todos estos Centros de Entrega Globales cuentan con certificación en altos niveles de calidad y servicio, así mismo, la empresa cuenta con un sistema interno de calidad, IQMS alineado a estándares internacionales como: ISO 9001, ISO 20000, ISO 27000, TL 9000, y a los modelos CMMI Nivel 5 y PCMM. Además la empresa opera cumpliendo los estándares más altos de infraestructura y seguridad física, de redes, del personal, y de sistemas [5].

Algunos de sus principales clientes son: Aeromexico, IMSS, Bank of America, GE, JCI, Ceridian, Verizon, Banco Santander, BAC Credomatic, Banco Interamericano de Desarrollo, Banamex y América Móvil, entre otros [5]. 
Tabla 3. Listado de certificaciones en el nivel 4 de CMMI-DEV ver. 1.3 año 2013, por país.

\begin{tabular}{c|cc}
\hline Posición & País & $\begin{array}{c}\text { Certificaciones } \\
\text { en Nivel 4 }\end{array}$ \\
\hline 1 & China & 26 \\
2 & Japón & 5 \\
3 & EEUU & 2 \\
3 & República & 2 \\
& de Corea & \\
4 & México & 1 \\
4 & Brasil & 1 \\
4 & Taiwán & 1 \\
\hline
\end{tabular}

En lo que respecta a certificaciones en el nivel 4 de madurez, China se encuentra en primer lugar con 26 certificaciones, le sigue Japón con 5, Estados Unidos y Republica de Corea con 2, y finalmente México, Brasil y Taiwán con 1 respectivamente [4]. La información se muestra en la Tabla 3.

En las estadísticas internacionales del nivel 3 de madurez en CMMI-DEV ver 1.3, el primer lugar lo ocupa India con 115 certificaciones, en segundo lugar le sigue México con 27, en tercer lugar con 18 certificaciones se encuentra Brasil, en cuarto lugar se encuentra República de Corea con 16 certificaciones y Japón ocupa el quinto lugar con 14 certificaciones [4], esto lo podemos observar en el listado de la Tabla 4.

Tabla 4. Listado de certificaciones en el nivel 3 de CMMI-DEV ver. 1.3 año 2013, por país.

\begin{tabular}{c|cc}
\hline Posición & País & $\begin{array}{c}\text { Certificaciones } \\
\text { Nivel 3 }\end{array}$ \\
\hline $\mathbf{1}$ & India & 115 \\
$\mathbf{2}$ & México & 27 \\
$\mathbf{3}$ & Brasil & 18 \\
$\mathbf{4}$ & República & 16 \\
& de Corea & \\
$\mathbf{5}$ & Japón & 14 \\
$\mathbf{6}$ & Turquía & 11 \\
$\mathbf{7}$ & España & 10 \\
\hline
\end{tabular}


En lo que corresponde al nivel 2 de madurez en CMMI-DEV ver 1.3, los primeros seis lugares corresponden a los siguientes países, el primer lugar en certificaciones es Estados Unidos con 72, seguido por España con 21, México con 17, Brasil con 14, Francia con 12 y República de Corea con 11 certificaciones [4], esto se muestra en la Tabla 5.

Tabla 5. Listado de certificaciones en el nivel 2 de CMMI-DEV ver. 1.3 año 2013, por país.

\begin{tabular}{c|cc}
\hline Posición & País & $\begin{array}{c}\text { Certificaciones } \\
\text { Nivel 2 }\end{array}$ \\
\hline 1 & EEUU & 72 \\
2 & España & 21 \\
3 & México & 17 \\
4 & Brasil & 14 \\
5 & Francia & 12 \\
6 & República & 11 \\
& de Corea & \\
\hline
\end{tabular}

4.1. Análisis estadístico de certificaciones en CMMI-DEV ver. 1.3 en México en el año 2013

La constante competencia en el mercado de desarrollo de software a nivel mundial, ha impactado considerablemente a las empresas mexicanas dedicadas a este rubro, motivándolas a mejorar sus procesos y sus productos de software. México no se queda atrás con respecto a la implantación del Modelo CMMI-DEV ver 1.3, como se puede apreciar en la Tabla 1, en la que México ocupó el cuarto lugar a nivel mundial en certificación en CMMI-DEV ver 1.3, en el año 2013.

A continuación se presentan en la Tabla 6 un listado de las empresas mexicanas certificadas en CMMI-DEV ver 1.3, que obtuvieron alguna certificación, el nivel de madurez que alcanzaron y el estado de la República Mexicana en el que están establecidas [4].

Tabla 6. Empresas Mexicanas Certificadas en CMMI-DEV ver. 1.3, año 2013.

\begin{tabular}{l|ll}
\hline \multicolumn{1}{c|}{ Nombre de la Empresa } & Nivel & Estado \\
\hline ECARESOFT MEXICO S.A. DE C.V. & 5 & Nuevo León \\
IBM & 5 & Jalisco \\
TATA CONSULTANCY SERVICES & 5 & Estado de México \\
LIMITED & 5 & Queretaro \\
TECNOCOM & 5 & \\
\hline
\end{tabular}




\begin{tabular}{|c|c|c|}
\hline $\begin{array}{l}\text { INNOVACIÓN INTELIGENTE S. DE } \\
\text { R.L. DE C.V. }\end{array}$ & 4 & Jalisco \\
\hline $\begin{array}{l}\text { APPLIED PROTOCOL } \\
\text { INTERFACES, S.A. DE C.V. }\end{array}$ & 3 & Sinaloa \\
\hline ASTECI S.A. DE C.V. & 3 & Estado de México \\
\hline AXXIS SOLUCIONES S.A. DE C.V. & 3 & Estado de México \\
\hline $\begin{array}{l}\text { AZERTIA TECNOLOGIAS DE LA } \\
\text { INFORMACIÓN MÉXICO, S.A. DE } \\
\text { C.V. UNA EMPRESA DEL GRUPO } \\
\text { INDRA }\end{array}$ & 3 & Estado de México \\
\hline BANCO BASE & 3 & Nuevo León \\
\hline BIOXOR S. DE R.L. DE C.V. & 3 & Jalisco \\
\hline BSD ENTERPRISE, S.A. DE C.V. & 3 & Nuevo León \\
\hline $\begin{array}{l}\text { CORPORACIÓN DE SERVICIOS EN } \\
\text { TECNOLOGÍAS DE LA } \\
\text { INFORMACIÓN S.A. DE C.V. }\end{array}$ & 3 & Estado de México \\
\hline $\begin{array}{l}\text { DELOITTE CONSULTING GROUP } \\
\text { S.C. }\end{array}$ & 3 & Estado de México \\
\hline EBCOMM S.A. DE C.V. & 3 & Nuevo León \\
\hline EC SERVICES, S.A. DE C.V. & 3 & Jalisco \\
\hline EMERGYS MÉXICO S.A. DE C.V. & 3 & Jalisco \\
\hline $\begin{array}{l}\text { HEWLETT PACKARD } \\
\text { ENTERPRISE SERVICES }\end{array}$ & 3 & Chihuahua \\
\hline HILDEBRANDO & 3 & Estado de México \\
\hline $\begin{array}{l}\text { IBM DE MEXICO, } \\
\text { COMERCIALIZACION Y } \\
\text { SERVICIOS, S. DE R.L. DE C.V. } \\
\text { INFOVIEWS S.A. DE C.V }\end{array}$ & 3 & $\begin{array}{l}\text { Ciudad de } \\
\text { México, D.F. } \\
\text { Estado de México }\end{array}$ \\
\hline $\begin{array}{l}\text { INSTITUTO TECNOLÓGICO } \\
\text { SUPERIOR DEL SUR DE } \\
\text { GUANAJUATO (ITSUR) } \\
\text { IT SKILL, S.A. DE C.V }\end{array}$ & 3 & Estado de México \\
\hline LUMINA AMÉRICAS S.A. & 3 & $\begin{array}{l}\text { Ciudad de } \\
\text { México, D.F. }\end{array}$ \\
\hline $\begin{array}{l}\text { SOFTNET SOLUCIONES, S.A. DE } \\
\text { C.V. }\end{array}$ & 3 & Nuevo León \\
\hline SOLUTIA INTELLIGENCE S.A. DE & 3 & Jalisco \\
\hline
\end{tabular}




\begin{tabular}{|c|c|c|}
\hline C.V. & & \\
\hline SYE SOFTWARE S.A. DE C.V. & 3 & Jalisco \\
\hline $\begin{array}{l}\text { TATA CONSULTANCY SERVICES } \\
\text { DE MÉXICO S.A. DE C.V. }\end{array}$ & 3 & Estado de México \\
\hline $\begin{array}{l}\text { TECNOLOGÍA DE GESTIÓN Y } \\
\text { COMUNICACIÓN S.A. DE C.V. }\end{array}$ & 3 & Chihuahua \\
\hline $\begin{array}{l}\text { UNIVERSIDAD AUTÓNOMA DE } \\
\text { NUEVO LEÓN }\end{array}$ & 3 & Nuevo León \\
\hline VEDASOLUTIONS PROVIDER, S.C. & 3 & Estado de México \\
\hline $\begin{array}{l}\text { WORLD SOFTWARE SERVICES } \\
\text { GROUP S.A. DE C.V. }\end{array}$ & 3 & Nuevo León \\
\hline ALDEASOFT & 2 & Sinaloa \\
\hline ALGORIA SOFTWARE, S.C. & 2 & Sinaloa \\
\hline AXA SEGUROS, S.A. DE C.V. & 2 & $\begin{array}{l}\text { Ciudad de } \\
\text { México, D. F. }\end{array}$ \\
\hline $\begin{array}{l}\text { CONECTIVIDAD Y } \\
\text { TELECOMUNICACIÓN, S.A. DE } \\
\text { C.V. }\end{array}$ & 2 & Guanajuato \\
\hline CONSULTORES PLASCENCIA SC. & 2 & Jalisco \\
\hline $\begin{array}{l}\text { DESPACHO DE ASESORÍA } \\
\text { FINANCIERA INTEGRAL S.C. }\end{array}$ & 2 & Sinaloa \\
\hline $\begin{array}{l}\text { DESARROLLOS MULTIMEDIALES } \\
\text { INTERACTIVOS, S.A. DE C.V. }\end{array}$ & 2 & Jalisco \\
\hline GB NETWORKS S.A. DE C.V. & 2 & Jalisco \\
\hline IB - MED S.A. DE C.V. & 2 & Jalisco \\
\hline INNOVAWEB SA DE CV & 2 & Sinaloa \\
\hline $\begin{array}{l}\text { MASSOLUCIONES SOFTWARE } \\
\text { S.A. DE C.V. } \\
\text { MESSOFT SYSTEMS S.A DE C.V. }\end{array}$ & 2 & $\begin{array}{l}\text { Guanajuato } \\
\text { Jalisco }\end{array}$ \\
\hline MINDTEC & 2 & Sinaloa \\
\hline $\begin{array}{l}\text { S\&C CONSTRUCTORES DE } \\
\text { SISTEMAS, S.A. DE C.V. } \\
\text { TEQUI SOFT TECH, SAPI DE C.V. }\end{array}$ & 2 & $\begin{array}{l}\text { Ciudad de } \\
\text { México, D.F. } \\
\text { Jalisco }\end{array}$ \\
\hline $\begin{array}{l}\text { WERNER PEGASUS, S. DE R.L. DE } \\
\text { C.V. }\end{array}$ & 2 & Jalisco \\
\hline ZONA HS, S.A. DE C.V. & 2 & Sinaloa \\
\hline
\end{tabular}


Como podemos observar las cuatro empresas certificadas en el nivel 5, se encuentran ubicadas en los estados de la República Méxica con mayor desarrollo económico, la empresa ECARESOFT MEXICO S.A. DE C.V., es una empresa mexicana de tecnología de información especializada en el sector salud, que se ubica en Nuevo León; también encontramos a IBM en Jalisco y desde luego como ya la habíamos mencionado a nivel internacional la empresa TATA CONSULTANCY SERVICES LIMITED, con su corporativo en nuestro país, en el Esatdo de México.

Finalmente también encontamos en el nivel 5 a la empresa TECNOCOM, es una multinacional española de las tres primeras empresas del sector de la tecnología de la información en España, su presencia regional incluye además de España y México, Portugal y otros países de América Latina (Chile, Colombia, Perú, Brasil, Paraguay y República Dominicana) y en EEUU, Miami.

De la Tabla 6, después de hacer un análisis discreto, podemos concluir que el estado con más empresas certificadas en CMMI-DEV ver. 1.3, es Jalisco con 14, le sigue el Estado de México con 11, Nuevo León con 7, al igual que Sinaloa, después se ubica en cuarto lugar con 4 certificaciones, el Distrito Federal (D.F.), con 3 se encuentra Guanajuato, con 2, Chihuahua y en última posición con 1 certificación ubicamos a Querétaro. Esta información estadística esta concentrada en la Tabla 7.

Tabla 7. Número de certificaciones en CMMI-DEV ver. 1.3 por Estados en la República Mexicana.

\begin{tabular}{l|c}
\hline \multicolumn{1}{c|}{ Estado } & Certificaciones \\
\hline Jalisco & 14 \\
Estado de México & 11 \\
Nuevo León & 7 \\
Sinaloa & 7 \\
Ciudad de México, & 4 \\
D.F. & 3 \\
Guanajuato & 2 \\
Chihuahua & 1 \\
Querétaro & $\mathbf{4 9}$ \\
$\quad$ Total &
\end{tabular}

\subsection{Análisis estadístico de certificaciones en CMMI-DEV ver. 1.3 a nivel mundial y México en el año 2014}


En el mes de agosto de 2014 se realizó el estudio sobre las certificaciones logradas de CMMI-DEV ver. 1.3, el cual arrojó, que en lo que va del año 2014, existen 725 certificaciones mundiales, los datos fueron obtenidos de la página oficial del CMMI Institute del "Published Apraisal Results List 2014" [6], y el estudio dio como resultado la Tabla 8 , donde se ubican los cuatro países con mayor número de certificaciones a nivel mundial, cabe destacar que México ocupa el tercer lugar mundial en certificaciones del modelo CMMI-DEV ver. 1.3 y el primer lugar lo ocupa China con 339 certificaciones de un total de 725 .

Tabla 8. Listado de Países con mayor número de Certificaciones CMMI-DEV Ver. 1.3, año 2014.

\begin{tabular}{c|r}
\hline País & Certificaciones \\
\hline China & 339 \\
E.U. & 121 \\
México & 120 \\
India & 82 \\
\hline
\end{tabular}

En lo que se refiere a México el estudio dio como resultado el resumen que se presenta para los años 2013 y 2014 en la Tabla 9, donde se concentran el número de certificaciones por nivel de madurez en los ultimos dos años.

Tabla 9. Listado de Certificaciones de empresas Mexicanas en CMMI-DEV ver. 1.3 año 2013 y 2014.

\begin{tabular}{c|rrrrc}
\hline Año & Nivel 2 & Nivel 3 & Nivel 4 & Nivel 5 & Total \\
\hline 2014 & 52 & 47 & 7 & 14 & 120 \\
2013 & 17 & 27 & 1 & 4 & 49 \\
\hline
\end{tabular}

Los datos obtenidos de las certificaciones del año 2014, fueron considerados hasta el 15 de agosto de 2014, y en comparativa con los datos obtenidos del año 2013, el crecimiento en Certificaciones CMMI-DEV Ver.1.3 ha sido importante, ya que en el año 2013 se lograron únicamente 49 certificaciones en los diferentes niveles de madurez y en el transcurso del año 2014 se tienen registradas 120 certificaciones, si esta tendencia continua, al finalizar el año México habrá obtenido aproximadamente 180 certificaciones y abra aumentado su certificaciones $250 \%$ más a comparación del año anterior.

Esto tiende a ser posible si consideramos que en este año deben ser evaluadas las empresas favorecidas por la Convocatoria PROSOFT 2013-1, 2013-2 [7,8], que a través 
del Gobierno Federal y de la Secretaría de Economía obtuvieron apoyos económicos para empresas que realizan actividades vinculadas al sector de tecnologías de información (TI), para: certificación de competencias; creación de empleo de alta especialización; proyecto productivo; certificación organizacional; vinculación academia-industría; innovación; comercialización, entre otros.

Muchas de las empresas mexicanas que han logrado alguna certificación en CMMI al menos del 2013 a la fecha, ha sido por medio de programas de financiamientos que ofrece el Gobierno Federal a través de la Secretaria de Economía, pues todo lo que implica obtener una certificación, desde la capacitación, la implementación del modelo y finalmente la evaluación tienen un alto costo sobre todo para PyMES (Pequeñas y Medianas Empresas), de tal forma que estos apoyos buscan fomentar la creación, desarrollo, consolidación, viabilidad, productividad, competitividad y sustentabilidad de las empresas del sector de tecnologías de información y servicios relacionados, dirigidos a crear una cultura de Calidad.

\section{Resumen de resultados}

En este artículo se presentó el estudio de certificaciones en CMMI-DEV ver 1.3 logradas por organizaciones de todo el mundo, estos resultados se agruparon en 53 países y nos dio como resultado el ranking mundial. Los resultados de la investigación incluyen el número de certificaciones por país en cada uno de los niveles de madurez, que van del nivel 2 al nivel 5, con los que podemos observar el gran avance que tiene el país de China en cuanto a madurez en sus organizaciones, ya que cuenta con 621 empresas certificadas, también se hace notoria la participación de Estados Unidos con 251, India con 155 y México 49.

Cabe resaltar que México obtuvo el primer lugar en lo que se refiere a países de América Latina en cuanto a certificaciones en CMMI-DEV ver.1.3 en el año 2013.

En comparativa con el año 2013, en lo que va del año 2014, México ha logrado 120 certificaciones, ocupando el tercer lugar a nivel mundial, en segundo lugar se encuentra India con 121, en primer lugar se encuentra China con un total de 339 certificaciones.

\section{Conclusiones}

Los resultados demuestran que cada día crece más el número de empresas a nivel mundial preocupadas por mejorar sus procesos y realizar productos de calidad, que les garanticen la preferencia y permanencia en el mercado, fue quizás inesperado el haber encontrado a México en el tercer lugar a nivel mundial con 120 certificaciones, sin duda ha aumentado considerablemente el número de empresas certificadas en CMMI, esto puede ser una consecuencia del apoyo que actualmente esta ofreciendo el Gobierno Federal a través de la Secretaría de Economía a las empresas desarrolladoras de Software, por ello es indispensable que las organizaciones no pierdan de vista la mejora sus 
procesos y la importancia de las Certificaciones Internacionales, para que México pueda seguir estableciéndose como punta de lanza a nivel mundial en cuanto a las certificaciones en CMMI. Es importante considerar ahora una evaluación sobre los resultados de los apoyos brindados por las convocatoria de la Secretaría de Economía a las empresas beneficiadas con la finalidad de conocer el Nivel de Madurez que alcanzarón, la Certificación obtenida, los problemas a los que se enfrentarón, como los resolvieron y las estrategías tomadas para lograr la Certificación.

\section{Referencias}

1. Velthuis, M. G., Rubio, F. O., Guzman, I. G., \& Pino, F.: Calidad de Sistemas de Informacion. Mexico, D.F. Alfa Omega RA-MA. (2012)

2. CMMI Institute: Reporte técnico CMMI-DEV VER. 1.3. 2010. [En línea]. disponible:http://cmmiinstitute.com/assets/Spanish\%20Technical\%20Report $\% 20 \mathrm{CM}$ MI\%20V\%201\%203.pdf. [Ultimo acceso:18 de febrero de 2014].

3. Clearmodel, C.I. CMMI Institute: Capability Madurity Model Integration. [En línea]. Disponible: http://cmmiinstitute.com/. 2014. [Último acceso: 15 Agosto 2014].

4. CMMI-Institute: Published Apraisal Results List, 2013. [En línea]. Disponible: https://sas.cmmiinstitute.com/pars/pars.aspx. [Último acceso: 16 enero 2014].

5. CMMI-Institute: Published Apraisal Results List. 2014. [En línea]. Disponible: https://sas.cmmiinstitute.com/pars/pars.aspx. [Último acceso: 15 Agosto 2014].

6. Limited, T. C.: TCS IT Services, Consulting and Business Solutions. 2014. [En línea]. Disponible: http://www.tcs.com/worldwide/es/es/mexico/Pages/default.aspx, America Latina, Mexico. [Último acceso: 11 abril 2014].

7. Secretaría de Economía. Prosoft 3.0: CONVOCATORIA PROSOFT 2013-1, 2013. [En línea]. Disponible: http://www.prosoft.economia.gob.mx/ro2013/ Convocatoria PROSOFT 20131a.pdf. [Último acceso: 15 Agosto 2014].

8. Secretaría de Economía. Prosoft 3.0: CONVOCATORIA PROSOFT 2013-2, 2013. [En línea]. Disponible: http://www.prosoft.economia.gob.mx/ro2013/ Convocatoria\%20PROSOFT\%202013-2.pdf. [Último acceso: 15 Agosto 2014]. 